\title{
Occupation Time Statistics in the Quenched Trap Model
}

\author{
S. Burov, E. Barkai \\ Department of Physics, Bar Ilan University, Ramat-Gan 52900 Israel
}

\begin{abstract}
We investigate the distribution of occupation times for a particle undergoing a random walk among random energy traps and in the presence of a deterministic potential field $U^{\text {det }}(x)$. When the distribution of energy traps is exponential with a width $T_{g}$ we find that the occupation time statistics behaves according to (i) the canonical Boltzmann theory when $T>T_{g}$, (ii) while for $T<T_{g}$ they are distributed according to the Lamperti distribution with the asymmetry of the distribution determined by the Boltzmann factor $\exp \left(-U^{\text {det }}(x) / T_{g}\right)$ with $T_{g}$ and not $T$ being the effective temperature. We explain how our results describe occupation times in other systems with quenched disorder, when the underlying partition function of the problem is a random variable distributed according to Lévy statistics.
\end{abstract}

PACS numbers: 05.20.-y,02.50-r,46.65.+g

Consider a one dimensional Brownian motion, in a binding deterministic potential field $U^{\operatorname{det}}(x)$. Following the trajectory of an individual particle one may determine the occupation time $t^{\text {Occ }}$, which is the time the particle spends in the domain $x_{1}<x<x_{2}$. In the limit of long measurement times $t$ the occupation fraction $\bar{p} \equiv t^{\text {Occ }} / t$ is given by Boltzmann statistics, assuming that the dynamics is ergodic

$$
\bar{p} \rightarrow \frac{\int_{x_{1}}^{x_{2}} e^{-\frac{U^{\operatorname{det}}(x)}{T}} \mathrm{~d} x}{Z},
$$

where $Z=\int_{-\infty}^{\infty} \exp \left[-U^{\mathrm{det}}(x) / T\right] \mathrm{d} x$ is the normalizing partition function and $T$ is the temperature. We see that the occupation fraction $\bar{p}$ does not depend on the details of the dynamics, for example on the diffusion constant or the initial conditions, which is of-course the strength and the generality of statistical mechanics. Majumdar and Comtet showed that when the potential energy is random the occupation fraction may exhibit large fluctuations from one sample of disorder to another, in particular in [1] the Sinai model was investigated. Generally, for disordered systems an important question is what is the distribution of the occupation fraction and its relation to the underlying disorder.

Here we investigate occupation time statistics using the well known trap model [2, 3]. Briefly the model introduced in the seventies, describes a random walk among traps with a random depths of energy traps, with a density of states which is exponentially distributed with a width $T_{g}$ (see details below). Such density of states leads to anomalous diffusion [4, 5], and aging [2, 5, 6, 7] when $T<T_{g}$. The model, in fact a family of models, was used to describe dynamics of many systems: transport of electrons in amorphous materials [8, 9, 10], single molecule pulling experiments [11], rheology of soft matter [12] e.g. emulsions, relaxation in glasses [2, 3, 13] and green fluorescent protein dynamics [14] to name a few. Recently the aging scenario of the trap model [2, 3], was justified rigorously using the random energy model as the starting point [15].

Bouchuad 2] introduced the concept of weak ergodicity breaking, in the context of the trap model, which implies the breakdown of Boltzmann's statistics when $T<T_{g}$. The goal of this paper is to define and investigate the rather strong deviations from Boltzmann's statistics in the quenched trap model. In particular we investigate the distribution of the occupation times for the quenched trap model quantifying the deviations from the standard canonical theory Eq. (11). At the end of the paper we will show how the main features of our theory can describe occupation times in several other models of quenched disorder, proving some generality of our results beyond the trap model.

Quenched Trap Model We consider a particle undergoing a one dimensional random walk on a quenched random energy landscape on a lattice. Lattice points are on $x=0, a, 2 a, \cdots, L$ where $a$ is the lattice spacing. On each lattice point a random energy $E_{x}$ is assigned, which is minus the energy of the particle on site $x$, so $E_{x}>0$ is the depth of a trap on site $x$. The traps energies $\left\{E_{x}\right\}$ are independent identically distributed random variables, with a common probability density function $(\mathrm{PDF}) \rho(E)=\left(1 / T_{g}\right) \exp \left(-E / T_{g}\right)$. Due to an interaction with a heat bath the particle may escape site $x$ and jump to one of its nearest neighbors. The average time it takes the particle to escape from site $x$ is given by Arrehhius law $\tau_{x}=\exp \left(E_{x} / T\right)$. Notice that small changes in $E_{x}$ leads to an exponential shift in $\tau_{x}$. In particular it is easy to show that the PDF of the waiting times is

$$
\psi(\tau)=\frac{T}{T_{g}} \tau^{-\left(1+\frac{T}{T_{g}}\right)} \quad \tau \geq 1
$$

so when $T<T_{g}$ the average waiting time diverges.

An additional bias is applied to the system. For example in transport processes this is a driving field, e.g. an external electric field (and see more details below). Let $q_{x}\left(1-q_{x}\right)$ be the probability of jumping left (right) from site $x$ respectively. The master equation for the 
population on site $x, P_{x}$ is

$$
\frac{\mathrm{d} P_{x}}{\mathrm{~d} t}=-\frac{1}{\tau_{x}} P_{x}+\frac{q_{x+1}}{\tau_{x+1}} P_{x+1}+\frac{1-q_{x-1}}{\tau_{x-1}} P_{x-1} .
$$

For non biased random walks $q_{x}=1 / 2$ while for uniformly biased random walks $q_{x} \neq 1 / 2$ is a constant. The boundary conditions are reflecting $q_{0}=0$ and $q_{L}=1$ though our main results are valid also for periodic boundary conditions.

The local bias $q_{x}$ is controlled by a deterministic potential field $U_{x}^{\text {det }}$, which in some cases is controlled by the experimentalist. It is usually assumed that detailed balance conditions holds so that the dynamics of the populations reaches thermal equilibrium described by Boltzmann's canonical ensemble. For the trap model this well known condition leads to

$$
\frac{q_{x}}{1-q_{x-1}}=\exp \left[-\frac{\left(U_{x-1}^{\mathrm{det}}-U_{x}^{\mathrm{det}}\right)}{T}\right] .
$$

For example if a constant driving force field $\mathcal{F}$ acts on the system $q_{x}=1 /[1+\exp (\mathcal{F} a / T)][6]$.

We consider a single realization of disorder in the thermodynamic limit where the measurement time $t \rightarrow \infty$ before the system size is made large. One can show that the equilibrium of populations is described by Boltzmann statistics, which is not surprising since we used the detailed balance condition. The total time the particle spends in the domain $x_{1} \leq x \leq L$ is the occupation time $t^{\text {Occ }}$. This domain is called the observation domain. Assuming that the process is ergodic we have the occupation fraction for a single disordered system

$$
\bar{p}=\frac{t^{\mathrm{Occ}}}{t} \rightarrow \frac{Z^{\mathrm{O}}}{Z^{\mathrm{O}}+Z^{\mathrm{NO}}}
$$

where

$$
Z^{\mathrm{O}}=\sum_{x=x_{1}}^{L} \exp \left[-\frac{\left(U_{x}^{\text {det }}-E_{x}\right)}{T}\right]
$$

is the partition function of the part of the system under observation and $Z^{\mathrm{NO}}=\sum_{x=0}^{x_{1}-a} \exp \left[-\left(U_{x}^{\text {det }}-E_{x}\right) / T\right]$ is the partition function of the rest of the system. The occupation fraction is a random variable which varies from one system to the other, the goal of this manuscript is to calculate its distribution. However first four comments are in place. (i) If we have only a single realization of disorder the occupation fraction is given by Boltzmann statistics, the question then is whether the occupation fraction a self averaging quantity. Namely is it reproducible in a second experiment when a different realization of disorder is investigated. More generally we have in mind the situation where one investigates many realizations of disorder, for each the occupation fraction is random and hence one may construct its distribution, this case corresponds in principle to single molecule experiments where one may track independently a large number of individual molecules each one interacting with a unique random environment [16]. (ii) We have assumed that for a single disordered system the dynamics is ergodic. This is so since we are considering the thermodynamic limit where the measurement time $t \rightarrow \infty$ first while maintaining a finite size of the system. For a finite system we always have a finite $E_{\max }$ which is the maximum of the random energies $\left\{E_{x}\right\}$, and hence there is always a long time $t$ which is much larger than $\exp \left(E_{\max } / T\right)$ after which the process is behaving according to the ergodic principle. (iii) The occupation time in Eq. (5) describes rather generally the occupation time of a particle in a random energy landscape and is not unique to the specific dynamics of the quenched trap model. For example we could add random barriers to the dynamics of the model which would not alter its equilibrium. (iv) Previous work 17. considered the occupation times of the continuous time random walk (CTRW) model (annealed model), unlike the quenched trap model in the CTRW model ergodicity is broken and the system is not spatially disordered.

From Eq. (5) we see that the distribution of the occupation fraction $\bar{p}$ is obtained in principle from the distributions of two independent random partition functions $Z^{\mathrm{O}}$ and $Z^{\mathrm{NO}}$. Let $G_{Z^{\mathrm{O}}}(z)$ and $G_{Z^{\mathrm{NO}}}(z)$ be the PDFs of $Z^{\mathrm{O}}$ and $Z^{\mathrm{NO}}$ respectively. Then the PDF of the occupation fraction $f(\bar{p})$ is found using Eq. (5)

$$
f(\bar{p})=\int_{0}^{\infty} \mathrm{d} z z G_{Z^{\mathrm{NO}}}[(1-\bar{p}) z] G_{Z^{\mathrm{O}}}(\bar{p} z) .
$$

We now consider the problem of finding $G_{Z^{\circ}}(z)$.

If the deterministic part of the field $U_{x}^{\text {det }}$ is a constant, Eq. (6) shows that $Z^{\mathrm{O}}$ is a sum of independent identically distributed random variables, and then Gauss-Lévy limit theorems apply. In contrast, when $U_{x}^{\text {det }}$ is not a constant then we are dealing with the problem of summation of non-identically distributed random variables and hence in what follows we modify the familiar limit theorems for the case under investigation.

Let $n$ be the number of lattice points in the interval $\left[x_{1}, L\right]$. We consider the scaled random variable $\tilde{Z}^{\mathrm{O}}=$ $Z^{\mathrm{O}} / n^{1 / \alpha}$ with $\alpha=T / T_{g}$ and $T<T_{g}$. The Laplace $z \rightarrow u$ transform of the PDF of $\tilde{Z}^{\mathrm{O}}$ is found using Eq. (6) and $\rho(E)$

$$
\hat{G}_{\tilde{Z} \mathrm{O}}(u)=\exp \left\{\sum_{x=x_{1}}^{L} \ln \left[\hat{\psi}\left(\frac{u e^{-\frac{U_{x}^{\mathrm{det}}}{T}}}{n^{1 / \alpha}}\right)\right]\right\}
$$

where $\hat{\psi}(u)=\int_{0}^{\infty} \exp (-u \tau) \psi(\tau) \mathrm{d} \tau$. We now consider the limit of large $n$. We use the small $u$ expansion

$$
\ln [\hat{\psi}(u)] \sim-A u^{\alpha}+\frac{\alpha}{1-\alpha} u+\cdots
$$


where $A=\alpha|\Gamma(-\alpha)|$ and from Eqs. (819) we find

$\hat{G}_{\tilde{Z}^{\mathrm{O}}}(u) \sim$
$\exp \left\{-\frac{A u^{\alpha}}{n} \sum_{x=x_{1}}^{L} e^{-\frac{U_{x}^{\mathrm{det} \alpha}}{T}}+\frac{\alpha u}{(1-\alpha) n^{1 / \alpha}} \sum_{x=x_{1}}^{L} e^{-\frac{U_{x}^{\mathrm{det}}}{T}}+\cdots\right\}$

In the continuum limit of $a \rightarrow 0 n \rightarrow \infty$ and $L-x_{1}=$ an remaining finite we may replace the summation with integration and find the stretched exponential

$$
\hat{G}_{\tilde{Z}^{\mathrm{O}}}(u) \sim \exp \left[-A \frac{\int_{x_{1}}^{L} e^{-\frac{U^{\mathrm{det}}(x)}{T_{g}}} \mathrm{~d} x}{L-x_{1}} u^{\alpha}\right],
$$

where $U^{\operatorname{det}}(x)$ is the deterministic field in the continuum limit. The inverse Laplace transform of Eq. (11) is the one sided Lévy stable law.

A similar calculation is made for $Z^{\mathrm{NO}}$. We invert the Laplace transform Eq. (11), switch back to the original variable $Z^{\mathrm{O}}$ instead of the scaled one $\tilde{Z}^{\mathrm{O}}$, and find using Eq. (7)

$$
f(\bar{p}) \sim \frac{1}{(\mathcal{R})^{1 / \alpha}} \int_{0}^{\infty} \mathrm{d} z z l_{\alpha}[(1-\bar{p}) z] l_{\alpha}\left[\frac{\bar{p} z}{(\mathcal{R})^{1 / \alpha}}\right]
$$

with

$$
\mathcal{R}=\frac{P_{B}\left(T_{g}\right)}{1-P_{B}\left(T_{g}\right)}
$$

In Eq. (12) $l_{\alpha}(z)$ is the one sided Lévy stable PDF whose Laplace pair is $\hat{l}_{\alpha}(u) \equiv \exp \left(-u^{\alpha}\right) . P_{B}\left(T_{g}\right)$ is Boltzmann's probability of finding the particle in the observation domain calculated using the deterministic field with a temperature $T_{g}$

$$
P_{B}\left(T_{g}\right)=\frac{\int_{x_{1}}^{L} \exp \left(-U^{\operatorname{det}}(x) / T_{g}\right) \mathrm{d} x}{Z\left(T_{g}\right)} .
$$

Solving the integral Eq. (12) we find the Lamperti [18] PDF

$f(\bar{p}) \sim \frac{\sin \pi \alpha}{\pi} \frac{\mathcal{R} \bar{p}^{\alpha-1}(1-\bar{p})^{\alpha-1}}{\mathcal{R}^{2}(1-\bar{p})^{2 \alpha}+\bar{p}^{2 \alpha}+2 \mathcal{R}(1-\bar{p})^{\alpha} \bar{p}^{\alpha} \cos \pi \alpha}$.

Eq. (13, 15) are the main results of this manuscript, soon to be discussed in detail, which are valid in the glassy phase $T<T_{g}$.

For $T>T_{g}$ and in the same limit we have the usual canonical behavior

$$
f(\bar{p}) \sim \delta\left(\bar{p}-P_{B}(T)\right) .
$$

Eq. (16) shows that when $T>T_{g}$ the disorder plays no role, indicating the reproducibility of Boltzmann's statistics Eq. (1), when the disorder is weak.
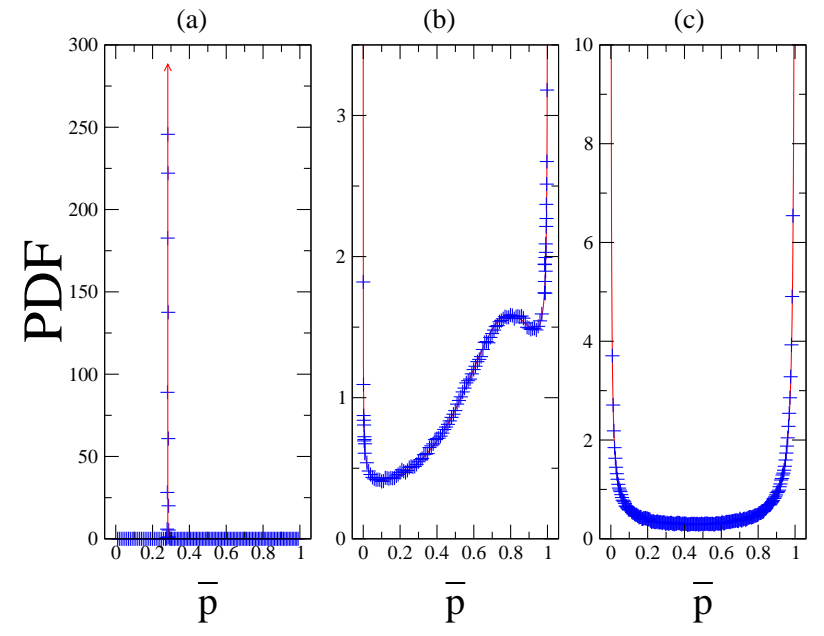

FIG. 1: The PDF of the occupation fraction for the deterministic field $U(x)=\mathcal{F} x$. (a) For $T=3 T_{g}$ we find a delta function centered on the value given by Boltzmann's statistics. When $T / T_{g}=0.7$ [panel (b)] the non trivial distribution of the occupation fraction has three peaks while for $T / T_{g}=0.3$ [panel (c)] the distribution is bi-modal. The + are simulations and the curve is the theoretical prediction Eq. (15) without fitting. We used $\mathcal{F}=1, T_{g}=1, a=10^{-5}$ and the observation domain $0<x<1$.

We now discuss the behaviors found in our main Eqs. (13, 15). These equations give the distribution of the occupation times, which is the generalization of the usual Boltzmann law Eq. (16). The parameter $\mathcal{R}$ is called the asymmetry parameter, and if $\mathcal{R}=1, f(\bar{p})$ is symmetric, for example when $T / T_{g}=1 / 2$ and $\mathcal{R}=1$ we get the arcsine PDF. The asymmetry parameter $\mathcal{R}$ is calculated by the usual type in integral over the Boltzmann factor, however now the temperature $T_{g}$ is the relevant temperature not $T$ [see Eq. (13)]. Roughly speaking there are two sources of fluctuations: the disorder characterized by $T_{g}$, and the temperature $T$. Hence when $T<T_{g}$ the relevant temperature is the "temperature of the disorder" that is $T_{g}$. For example using Eq. 13[15]16 the average occupation fraction has the following surprising discontinuous behavior,

$$
\langle\bar{p}\rangle=\left\{\begin{array}{cc}
P_{B}\left(T_{g}\right) & T<T_{g} \\
P_{B}(T) & T>T_{g} .
\end{array}\right.
$$

The average occupation fraction freezes in the colder glassy phase of $T<T_{g}$ in the sense that it does not depend on the temperature $T$, for any type of deterministic binding field.

In Fig. 1] we demonstrate our results comparing our theory with numerical simulations on a lattice. We consider the situation where the deterministic field is $U^{\operatorname{det}}(x)=\mathcal{F} x$ and $0<x$, and the observation domain is 
$0<x<T_{g} / \mathcal{F}$. In Fig. 1(a) with $T>T_{g}$ we see that the distribution of occupation fraction is very narrow with $\bar{p}=P_{B}\left(T=3 T_{g}\right)=1-e^{-1 / 3}$ indicating that the disorder is not important. In contrast when $T<T_{g}$ the behavior of the occupation fraction changes dramatically and $\bar{p}$ is non self averaging and random.

Eq. (15) shows that when $T / T_{g} \ll 1$ the PDF of occupation fraction is essentially composed of two delta functions centered on $\bar{p}=1$ and $\bar{p}=0$. Namely for some samples of disorder the particle is within the observation zone during all the observation time $t(\bar{p}=1)$ and in other samples the particle is never in the observation zone $(\bar{p}=0)$. This behavior is easy to understand when $T \rightarrow 0$ the minimum of the random potential energy is the most populated, and this minimum can be found either in the observation zone or out of it. As shown in Fig. 1.(c), for small but finite $T$ we have a non-trivial bi-modal $U$ shape of the PDF, which reflects this low temperature behavior. As the temperature increases we start seeing a third peak in the PDF of the occupation fraction being developed close to the ensemble average [see center peak in Fig. 1 (b)], so when $T \rightarrow T_{g}$ the self-averaging phase is approached.

In Fig. 2 we show the averaged occupation fraction versus temperature $T$ using the same deterministic potential field as in Fig. 1. For $T>T_{g}$ the average occupation fraction is $P_{B}(T)$ and hence as the temperature is decreased the average occupation time increases, since the particles condensate closer to the minimum of the deterministic field which is on $x=0$ as the temperature is reduced. However when $T=T_{g}$ we see in Fig. 2 a type of phase transition in the behavior of the averaged occupation fraction, and it does not depend on the temperature when $T<T_{g}$, as predicted by our theory Eq. (17). We note that the convergence of the numerical results to the exact ones derived in this paper turned out to be relatively slow close to the temperature $T=T_{g}$ if compared with temperatures far from $T_{g}$.

Finally let us discuss the generality of our results beyond the quenched trap model. We have divided our system into two, the observation domain and the rest of the system. The partition functions of these domains are random variables, due to the randomness of the underlying Hamiltonian. As we showed, one key ingredient of our theory is that the PDFs of these two partition functions are one sided Lévy stable PDFs. In that case we proved that our main Eq. (15) describes the statistics of occupation time. We know that Lévy statistics is common in Physics and well established mathematically, and hence it seems to us natural to expect that partition functions of random systems may have a Lévy distribution. Indeed a partition function is a sum over energy states and if these states are random the connection of the distribution of partition functions with limit theorems of sums of random variables is expected to be general. In particular we could show that our main results describe also occu-

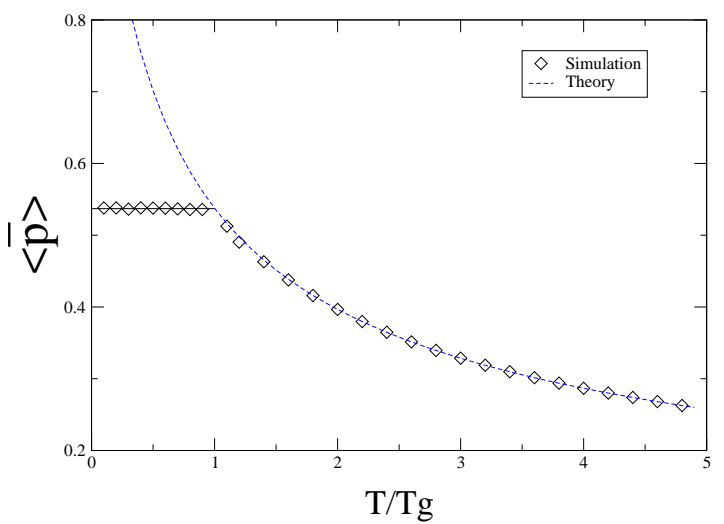

FIG. 2: The averaged occupation fraction versus $T / T_{g}$. When $T>T_{g},\langle\bar{p}\rangle=P_{B}(T)$ namely usual Boltzmann theory applies, while for $T<T_{g},\langle\bar{p}\rangle=P_{B}\left(T_{g}\right)$ which is independent of the temperature $T$. The lines are theoretical predictions Eq. (17) and the diamonds are simulation results with no fitting.

pation time statistics in several other models of quenched disorder: random comb model which is a simple model of a random walk on a loop-less random fractal [19], or models of anomalous diffusion of a particle on structures with distributed dangling bonds in the presence of bias [20, 21, 22]. Unlike the trap model, in these models geometry is the main factor responsible for the non-trivial occupation fraction. Sure the exponent does not generally turn out to be $\alpha=T / T_{g}$ as in the quenched trap model, but our main results are valid as we will discuss in a longer publication.

Acknowledgment This work was supported by the Israel Science Foundation. EB thanks A. Comtet, S. Majumdar, and G. Margolin for discussions.

[1] S. N. Majumdar and A. Comtet Phys. Rev. Lett. 89 060601 (2002).

[2] J. P. Bouchaud J. De Physique I 21705 (1992).

[3] J. P. Bouchaud and D. S. Dean J. Phys. I France 5265 (1995).

[4] E. M. Bertin and J. P. Bouchaud, Phys. Rev. E67 026128 (2003).

[5] C. Monthus and J. P. Bouchaud J. Phys. A 293847 (1996).

[6] E. M. Bertin and J. P. Bouchaud, Phys. Rev. E 67, 065105(R) (2003).

[7] B. Rinn, P. Maass, and J. P. Bouchaud Phys. Rev. Lett. 845403 (2000).

[8] M. Silver and L. Cohen Phys. Rev. B 15, 32763278 (1977).

[9] M. Silver, G. Schoenherr, and H. Baessler Phys. Rev. Lett. 48352 (1982).

[10] J. Orenstein and M. Kastner, Phys. Rev. Lett. 461421 (1981). 
[11] J. Brujic, et al Nature Physics 2282 (2006).

[12] P. Sollich, F. Lequeux, P. Hebraud, and M. E. Cates Phys. Rev. Lett. 782020 (1997).

[13] V. Bercu et al J. Phys. Cond. Matter 16 L479 (2004).

[14] P. Didier, L. Guidoni, and F. Bardou, Phys. Rev. Lett. 95090602 (2005).

[15] G. Ben Arous, A. Bovier, and V. Gayrand, Phys. Rev. Lett. 88087201 (2002).

[16] E. Barkai, Y. Jung, and R. Silbey Annual Review of Physical Chemistry 55, 457 (2004).

[17] G. Bel and E. Barkai Phys. Rev. Lett. 94240602 (2005).
[18] J. Lamperti, Trans. Amer. Math. Soc. 88380 (1958).

[19] D. ben-Avraham and S. Havlin, Diffusion and Reactions in Fractals and Disordered Systems Cambridge University Press (2000).

[20] J.P. Bouchaud and A. Georges, Physics Reports 195127 (1990).

[21] M. Barma and D. Dhar J. Phys. C 161451 (1983).

[22] A. Bunde, S. Havlin, H. E. Stanley, B. Trus, and G. Weiss Phys. Rev. B 348129 (1986). 